\title{
Temperature monitoring on a plasmatron experiment by pyroreflectometry
}

\author{
by R. Gilblas*, T. Sentenac*, D. Hernandez**, O. Chazot***, and Y.Le Maoult* \\ * ICA (Institut Clément Ader), Toulouse University, Mines Albi, Campus Jarlard, F-81013 Albi cedex 09, France, \\ rgilblas@mines-albi.fr \\ **Processes, Materials and Solar Energy (PROMES), CNRS, B.P.5, 66125 Font-Romeu Cedex, France \\ ${ }^{\star * \star}$ Environmental and Applied Fluid Dynamics Department, Von Karman Institute, 72 Chausse de Waterloo,
} Rhode-St-Genèse, 1640, Belgium

\begin{abstract}
In the domain of re-entry materials submitted to plasma heating, the temperature reached by the samples is a prior information. In this paper, the capability of pyroreflectometry [1] to measure the true surface temperature of a C-SiC sample exposed to the high-enthalpy plasma of the Plasmatron facility [2] is assessed. This study includes general thoughts about the influence quantities on the measurement : emissivity variations during the experiment, plasma emission and windows transmission. We propose then a method called pyroreflectometry, and we calibrated the system in a way to solve the three previous problems. In the first part, the Plasmatron facility and the pyroreflectometry method are presented. Then, the radiative properties of the scene are retrieved and/or measured by spectrometry. Finally, the results of pyroreflectometry on the Plasmatron facility are displayed and commented. We conclude that this first instrumentation is very encouraging, the true temperature of the sample is retrieved and the ablation signature through the emissivity variations is appearing.
\end{abstract}

1.

Introduction

Nowadays, in the space domain, when a satellite is launched, important issues concern its destiny off service. One approach is to get back the spacecraft after its return on Earth, so this latter must be protected during its atmosphere re-entry. To do so, the spacecraft must be equipped with shield materials, which goal is to burn entirely in the atmosphere. This problematic raises scientific issues, and material scientists have to test re-entry atmosphere materials to evaluate their abilities [3]. Obviously, the temperature of the samples tested is a prior information for the analysis of the data.

Plasma studies enable the simulation of re-entry conditions in the atmosphere in terms of flows (composition, velocities and energies) and temperatures [2]. When a sample is submitted to a plasma torch, some high energy phenomenon can occur like oxidation or ablation [4]. In this article, a C-SiC composite is exposed to a high energy plasma. Ablation happens and the layers constituting the sample are ejected out one after the other. This experiment is then critical for non contact temperature measurement like pyrometers : the emissivity is the Sic's one on the beginning of the trial and can become Carbon's one suddenly when a layer is ablated. We will then present a method which helps to treat those emissivity discontinuity.

In the field of non contact temperature measurements, the main causes of errors are the spectral settings and the hypothesis. About the first point, it would be wise to work in the near-infrared domain or in the visible domain because the temperature reached can be very high (about $2500^{\circ} \mathrm{C}$ ), so the sensibility will be maximum. The second point (hypothesis) has different impacts, depending on the material under test. Monochromatic pyrometry [5] supposes that the emissivity is known, and the eventual variations with time can be corrected by post treatment. It is obviously difficult to perform on-line, when an unknown phenomenon occurs. Bichromatic pyrometry [6] supposes that the emissivity ratio at two near wavelengths is known and constant. This hypothesis is wrong is the majority of the cases, and leads to large errors on temperature. Finally, multiwavelength pyrometry [7] is more complete because it modelizes the emissivity variation like a polynomial or exponential model versus wavelength. However, for this experiment where the material changes with temperature, no model can be adapted for those two materials which have very different emissivity spectrum.

To avoid the problem of emissivity discontinuity with temperature (and/or time) and wavelength, the idea would be to propose an active method which measures simultaneously emissivity and temperature. In this article, we present a monitoring by pyroreflectometry [1], which performs an indirect measurement of emissivity by the measurement of bidirectional reflectivities at two wavelengths. In addition to the emissivity discontinuity problem, two other problems are discussed and treated: the plasma influence on the measurement and the windows transmission.

In this paper, we will first present the Plasmatron facility and the difficulties to measure an accurate temperature on it with non-contact method. Then, the radiative properties of the scene will be given, including emission and reflexion properties of the sample, and the plasma ones. Finally, first results of temperature monitoring on this experiment will be provided and analyzed. 
http://dx.doi.org/10.21611/qirt.2014.084

2. Temperature measurement problematic and proposition

In this section, the Plasmatron facility is presented, and the theoretical basis of pyroreflectometry are given.

\subsection{Presentation of the facility and temperature measurement problem}

The Plasmatron facility enables the generation of an inductively coupled plasma thanks to a high voltage, high frequency and high power source [2]. The ionized gas is air, and the heat flux generated in this high enthalpy plasma reach 1 to $351 \mathrm{~W} / \mathrm{m}^{2}$, which reproduces the conditions of atmosphere re-entry for spacecrafts. The pressure in the chamber is maintained between 6 and 12 mbar. The samples are inserted in the plasma torch, as depicted by figure 1 , and its temperature can quickly ramp up to $2000^{\circ} \mathrm{C}$, depending on its thermal properties and the plasma torch power.

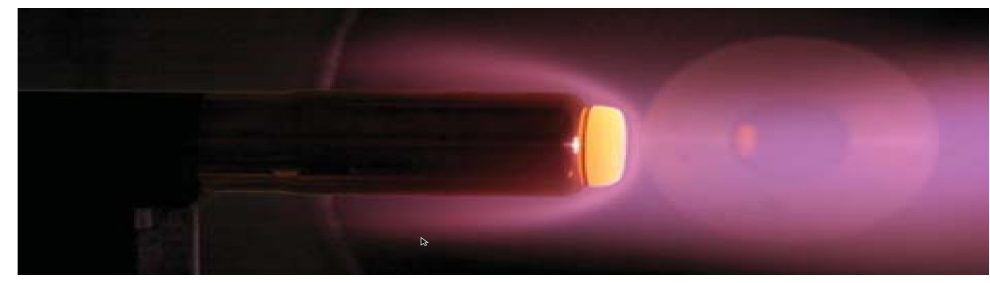

Fig. 1. Photograph of a sample submitted ti the plasma torch

A schematic drawing of the facility is given in figure 2, and helps to locate the windows for the non contact control and measurement. 5 quartz windows are located at one side of the chamber. The plasma torch generator is located at the centre of this side, so symmetrical conditions are obtained. The conditions of visualization of the sample are extreme, because viewing angles are high (about $45^{\circ}$ ), so directional effects may occur.
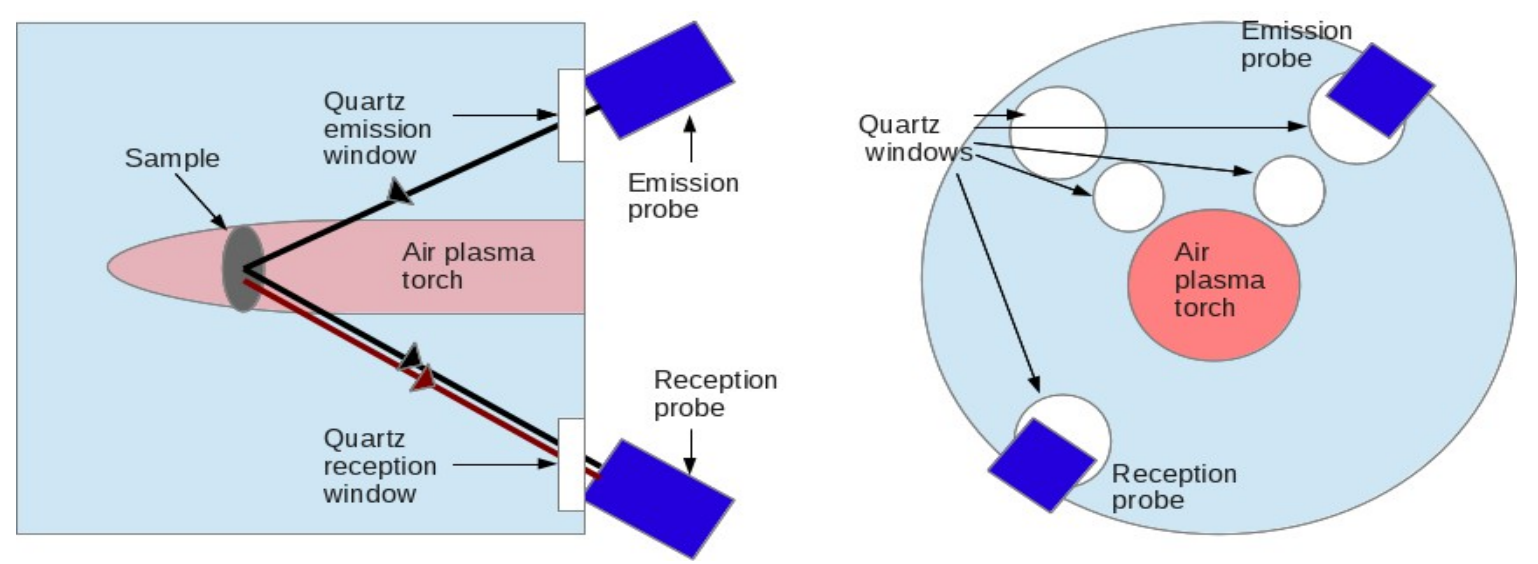

Fig. 2. Schematic view of the Plasmatron facility (right and front view)

About the temperature measurement, a detector located at one window in direction $r$ receives a radiance $L^{r}$ given by equation 1 for an opaque sample and with a negligible environment transmission.

$$
L^{r}(\lambda, T)=L^{0}\left(\lambda, T_{L}^{r}\right)=\tau_{w}(\lambda)\left(\varepsilon^{r}(\lambda) L^{0}(\lambda, T)+L_{p}^{r}\left(\lambda, T_{p}\right)+\left(1-\varepsilon^{r}(\lambda)\right) L_{p}^{r}\left(\lambda, T_{p}\right)\right)
$$

With : $L^{0}$ : black body radiance $; T_{L}$ : radiance temperature $; \tau_{w}$ : transmission of the window ; $\varepsilon_{r}$ : directional emissivity of the sample ; $T$ : temperature of the sample's surface; $L_{p}$ : plasma radiance $; T_{p}$ : plasma temperature.

This equation shows the main problematic for an accurate temperature measurement. Even if radiance temperature is measured by inverting the radiometric model and without considering the emissivity value, it is necessary to know several parameters to reach a good accuracy : 1- Knowledge of emissivity on-line to compensate the discontinuity caused by ablation. 2- Influence of Plasma emission, direct or reflected by the sample, and received by the detector. 3Transmission of the windows. Even if this latter is often considered as perfect, precautions are necessary when material weight losses occur and are possibly sources of pollution. 


\subsection{Theoretical basis of pyroreflectometry}

The proposition of temperature monitoring by pyroreflectometry addresses the three previous problems and tries to solve them.

1- About the emissivity value, the principle of pyroreflectometry enables to take it into account. Pyroreflectometry is a bichromatic method, which includes an indirect measurement of emissivity by reflectometry. In a first step, radiance temperatures $T_{\mathrm{L}}$, which are dependant on both temperature and emissivity, are measured at two wavelengths. Then, the bidirectional reflectivity $\rho$ is also measured and brings information about the emissivity. Finally, writing the radiometric equations at two wavelengths, it is possible to calculate the true temperature $\mathrm{T}$, corrected from the emissivity influence (see Equ.(2)). To do so, the material must satisfy a condition : the distribution of the reflectivites over the upper hemisphere must be the same for the two wavelengths considered. In other words, the diffusion factor $\eta$ must be invariant versus wavelength.

$$
\frac{1}{T}=\frac{1}{T_{L 1}}+\frac{C_{2}}{\lambda_{1}} \ln \left(1-\eta \rho_{1}\right) \quad \frac{1}{T}=\frac{1}{T_{L 2}}+\frac{C_{2}}{\lambda_{2}} \ln \left(1-\eta \rho_{2}\right)
$$

with $\mathrm{C}_{2}$ : Second Planck's constant

2- About the plasma emission, the wavelengths chosen for the method are located out of any peek of the air plasma. The radiance emitted becomes then negligible : $\quad\left(1-\varepsilon^{r}\right) L_{p}{ }^{r}<L_{p}^{r}<<\varepsilon^{r} L^{0}(\lambda, T)$. This result is detailed on next part.

3- For the transmission of the windows, the thermal calibration is performed with a black body and the window. To do so, the transmission of the window is included in the radiometric model coefficients.

\subsection{Apparatus and calibrations}

The pyroreflectometer is composed of two InGaAs detectors operating in the near-infrared domain and set on wavelengths 1,31 and $1,55 \mu \mathrm{m}$ via $100 \mathrm{~nm}$ spectral bandwidth filters. For the active phase, two $120 \mathrm{~mW}$ lasers at the same previous wavelengths are pulsed at $1 \mathrm{~Hz}$ and illuminate the sample via optical fibres and lenses with an area of about $1 \mathrm{~cm}^{2}$. Two windows of the Plasmatron are occupied by lenses, one for laser emission, and the other dedicated to reception signals. The commands and calculations are performed with a dedicated software and PC.

For the measurement of radiance temperature $T_{L}$, the thermal calibration is performed with a high temperature $[500-3000]^{\circ} \mathrm{C}$ blackbody available at the PROMES laboratory. An adjustment of gains and fibres diameters has been necessary to prevent from the detector's saturation and to still have a maximum SNR. As the sample is a bad reflector, the gains must be maximum to have a good reflectivity signals. But with this gains, the thermal signal saturates prematurely, so the thermal range is reduced to $[1000-2000]^{\circ} \mathrm{C}$.

About the reflectometric calibration, enabling the measurement of bidirectional reflectivities, we chose to perform an in-situ calibration in the Plasmatron facility. To do so, we replaced the sample on its sample holder by a Spectralon reflectivity standard and we measured the signal detected by the reception detector. As the model is linear, it is sufficient to calibrate this measurement.

3.

Radiative properties of the studied scene

In this part, the determination of all influence quantities appearing equation 1 is presented.

\subsection{Radiative properties of the plasma}

As explained before, if a system is based on the measurement of the sample's signal in the spectral bands defined by the filters used, high errors can be committed if the plasma is emitting in the same spectral bands. In our case, this signal variations will be interpreted as radiance temperature or bidirectional reflectivity variation, which is an artifact. So, we have to ensure that the plasma doesn't emit in our spectral bands, or, to a lesser degree, that it emits with the same intensity for the two wavelengths.

The radiative properties of the plasma are difficult to find for the near-infrared domain, however, reference [8] gives an overview of peeks appearing in this domain. They are superimposed on a continuous background made of thermal emission of the constituting gas. The main conclusion is that no peek seems to appear in our spectral band and the background seems equal for the two bands, so the temperature measurement shouldn't be impacted.

\subsection{Transmission of windows}

The windows are $2 \mathrm{~cm}$ thick quartz and $5 \mathrm{~cm}$ in diameter. Even if the transmission of the window is included is the radiometric coefficient, we still will measure it thanks to FTIR spectrometer. The values given table 1 shows that the 
quartz window is not transmitting identically for the two wavelengths. This phenomenon could be explain by a previous pollution of the windows, a thin layer could have been deposit on the window. To conclude, no error should be noticed on the temperature measured because thermal calibration is performed with it, but the transmission must not vary during the experiment.

\begin{tabular}{|l|l|l|}
\hline Transmission & $\lambda=1,31 \mu \mathrm{m}$ & $\lambda=1,55 \mu \mathrm{m}$ \\
\hline Reception window & 0,341 & 0,485 \\
\hline Emission window & 0,298 & 0,458 \\
\hline
\end{tabular}

Tab. 1. Transmission of the Quartz windows

\subsection{Radiative properties of the C/SiC Sample}

The sample tested in this experiment is a C-SiC composite, appearing fig.4, on the left. It is $2 \mathrm{~cm}$ in diameter and $2 \mathrm{~mm}$ thick. About its elaboration, some carbon fibres are woven and the sample is then covered with a SiC layer. The sample presents then a grey aspect due to the SiC deposition and the fibres are clearly visible and should induce some strong directional effects.

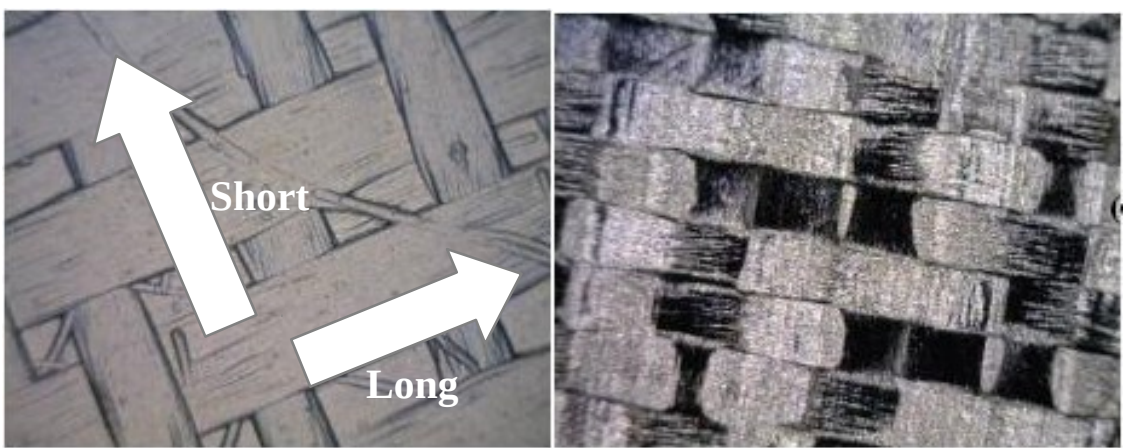

Fig. 3. Photograph of the sample before and after the trial (magnification $\times 50$ )

\subsubsection{Emission properties of the sample}

The FTIR spectrometer is equipped with an Infragold integrated sphere and enables the measurement of reflectance at room temperature. For opaque materials, the sum of reflectance with emissivity is equal to one. Two detectors are used : a DlaTGS for the [3-24] $\mu \mathrm{m}$ range and an InGaAs for the [0.9-2] $\mu \mathrm{m}$ range. The emissivity spectra of the sample is provided in figure 4.

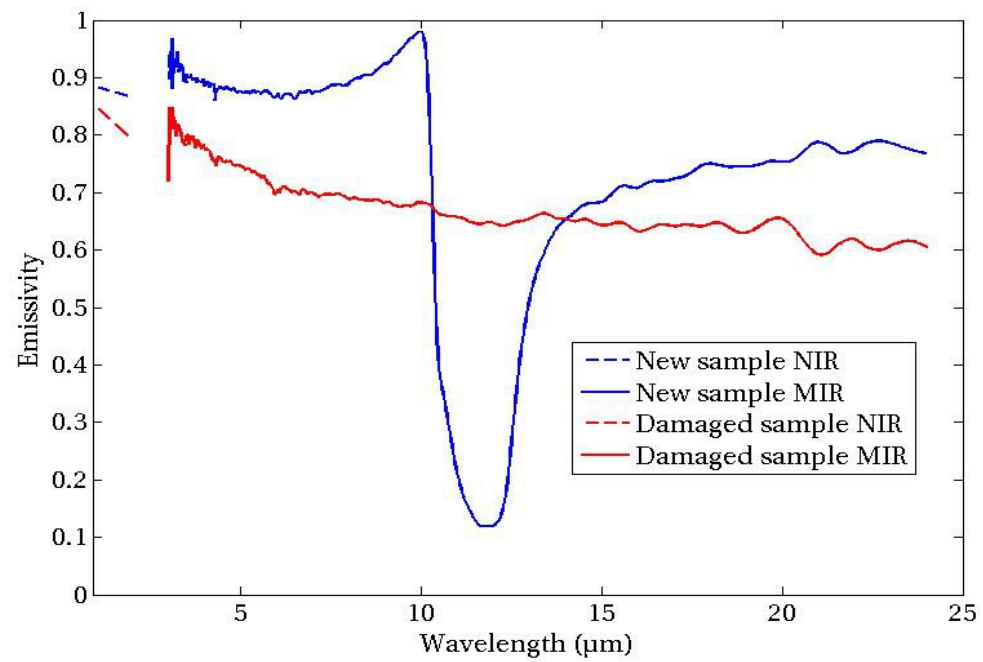

Fig. 4. Emissivity spectrum of the sample before and after trial 
The emissivity of the sample is high (between 0,9 to 1 ) from 1 to $10 \mu \mathrm{m}$. It decreases versus wavelength before $6 \mu \mathrm{m}$ and then increases to reach 1 at $10 \mu \mathrm{m}$. We recognize here the Christiansen's wavelength [9] of SiC, where emissivity is equal to one and almost doesn't vary with temperature. This point is then a very good repositioning wavelength for temperature measurement. After this, a band of low emissivity appears, and between 15 and $25 \mu \mathrm{m}$, emissivity is stable around 0.7 value. The damaged sample presents a monotonous decreasing emissivity, signature of the carbon fibres.

\subsubsection{Reflexion properties of the sample}

The spectrometer equipped with a goniometer enables the measurement of BRDF. Fixing the incidence angle to $45^{\circ}$ and varying the reception angle from 13 to $89^{\circ}$, it is possible to draw the distribution of the reflectivities for two NIR wavelengths. The result is shown figure 6 , for the two orientations of the sample depicted in figure 4.

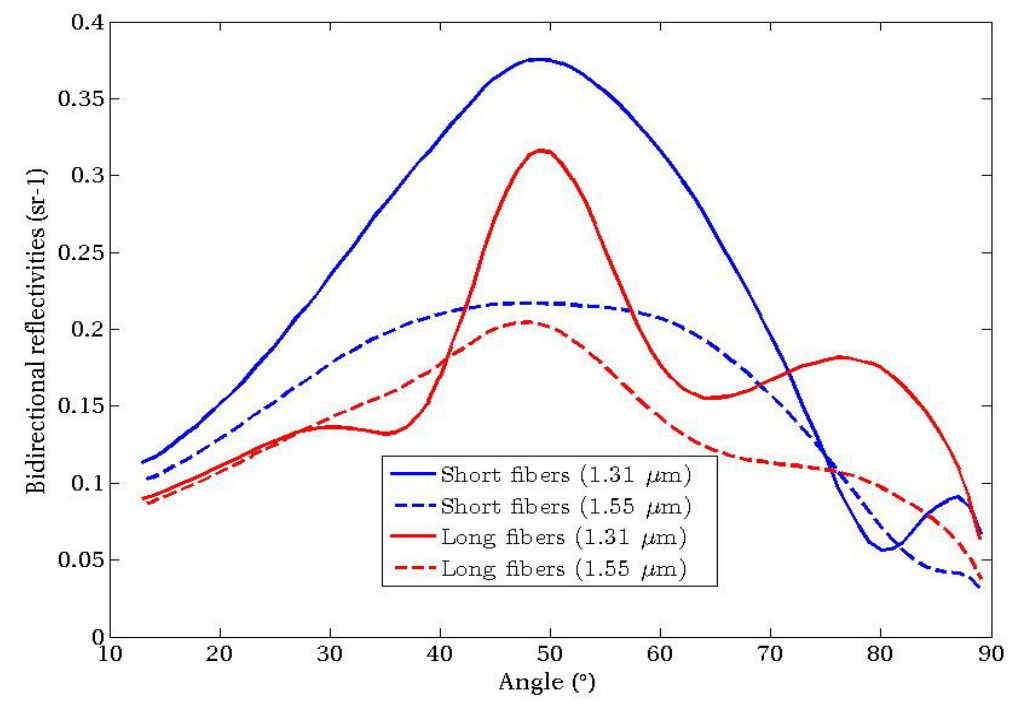

Fig. 5. BRDF of the sample versus the reception angle for two sample's orientation

The major conclusion is that the fibres have a strong influence on the reflected flux. In the short fibres orientation, the reflected flux is well diffused. On the other hand, for the long fibres orientation, some areas show sudden reflectivity drops. Still, we notice that the Snell-Descartes conditions induce a maximum reflected flux so are compatible with the conditions of the Plasmatron. Moreover, as the spot size is about $1 \mathrm{~cm}^{2}$, it illuminates several fibres so should 
lead to less texture-dependent radiative properties. Another important conclusion is to notice that reflectivity shapes for the two wavelengths look a lot alike. This result reinforces the pyroreflectometry hypothesis of the diffusion factor spectral invariance.

\section{Results of pyroreflectometry on the Plasmatron facility}

Once the vacuum is set in the chamber, the plasma torch is activated and the sample is inserted in the nozzle. Its temperature suddenly increases and the pyroreflectometer records the temperature variations and the evolution of the surface.

\subsection{True temperature results}

The measurements of the method (radiance temperature and bidirectional reflectivities) and the true temperature retrieved are shown in figure 6.

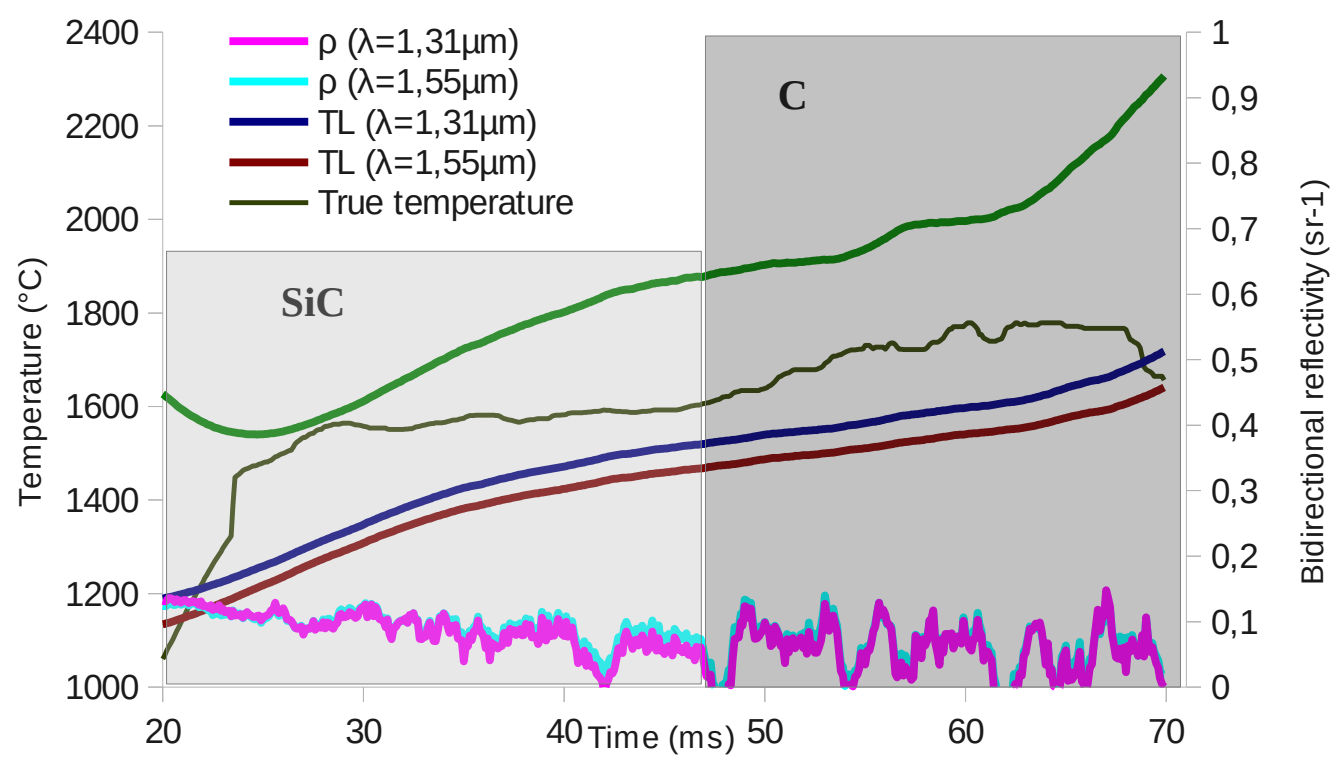

Fig.6. Results of pyroreflectometry

First of all, radiance temperatures are always well separated and wavelength $1.31 \mu \mathrm{m}$ is higher than $1.55 \mu \mathrm{m}$, as emissivity is. As the temperature increases, the two radiance temperatures go off. Supposing that the sample is grey, the calculation of bichromatic temperature is possible and is also displayed figure 6 . This latter globally over estimates the temperature, as emissivity decreases with wavelength. It is however a rather good approximation around $30 \mathrm{~ms}$, where the behavior is the SiC's one, almost grey.

The reflectivities are very noisy, this phenomenon may be due to the plasma influence on the laser illumination, or on the receiving quartz window. This phenomenon doesn't perturb the measurement as long as the reflectivity ratio at the two wavelength is kept constant. The general trend for reflectivities is a global decrease with temperature.

Combining those two previous analysis, the conclusion is that a surface state variation is occurring. Indeed, the post-mortem photograph of the sample shown figure 3 reveals the apparition of intern carbon fibres with a different weaving. The thickness before and after the trial is $2 \mathrm{~mm}$ and $1,4 \mathrm{~mm}$. The radiative properties measured by spectrometry and shown figure 4 clearly state that the carbon governs now the emission. So, when the temperature increases, the influence of carbon becomes more and more important compared to SiC. The sample becomes less emissive and less "grey". That is why bichromatic temperature starts to diverge when ablation starts.

The apparition of deep carbon fibres layer can also explain why reflectivity is perturbed during the experiment. Indeed, as each carbon fibre layer has its own weaving, the directional effect of reflectivity explained figure 5 should influence the bidirectional reflectivity.

Radiance temperatures and bidirectional reflectivities enable the calculation of true temperature. We see in figure 7 that it is still influenced by the plasma but its mean value, in dashed line, is very consistent with radiance temperature and bichromatic temperature. As it makes no hypothesis about the emissivity, it is more precise than bichromatic temperature and a deviation of $425^{\circ} \mathrm{C}$ is noticed at the end of the trial. 


\subsection{Emissivity calculation}

Once the true temperature is retrieved, it is possible to display the emissivity variations during the trial. It is shown in figure 7.

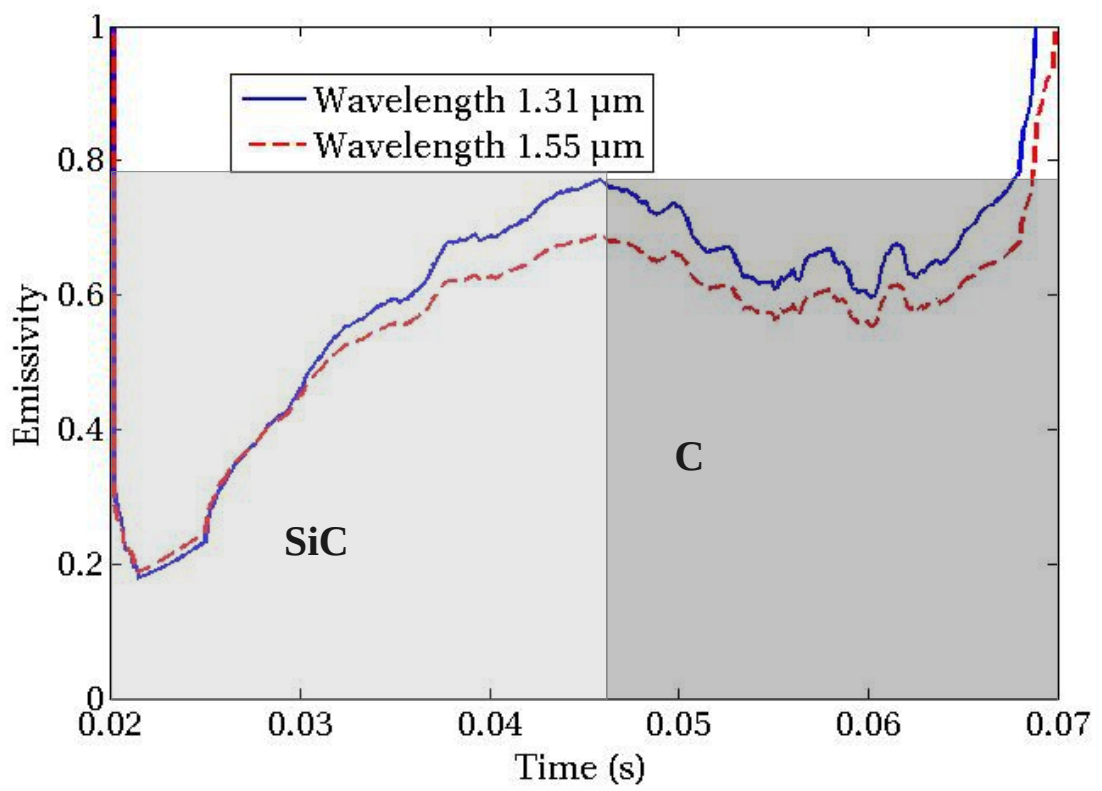

Fig. 7. Emissivity variations retrieved by pyroreflectometry

The emissivity variations calculated by pyroreflectometry reveals a global increase with temperature. The values found at the beginning of the trial are very low compared to spectrometry results. This incoherence can be explained by the noisy reflectivity signals, which is currently under corrections with the prospects given in next part.

General trends can be analyzed yet. Before $45 \mathrm{~ms}$, emissivity is increasing with time or temperature, starts with a grey behaviour $(\mathrm{SiC})$ and finish with non grey (Carbon). Then, at $45 \mathrm{~ms}$, the emissivity starts to drop and increases again after. This phenomenon can be attributed to the carbon fibres influence, which are less emissive than SiC for the same temperature. We can then suppose that we have on this curve a signature of ablation around $1500^{\circ} \mathrm{C}$, emphasized by the emissivity local thermal variation. Then the emissivity increases again, as temperature does.

5.

\section{Conclusion}

This paper presented the results of a temperature monitoring by an innovating non contact method called pyroreflectometry of the complex Plasmatron facility. The difficulty of this measurement was separated in three phenomenon : 1-Perturbations due to the plasma, 2- Non neutrality of viewing windows and 3-Variations of surface state during the trial. The results presented here are very encouraging and opens interesting prospects in terms of temperature measurement and physical analysis of re-entry materials.

First of all, we presented the problematic of the measurement and the necessity to have an on-line knowledge of emissivity in dynamical conditions. In addition to this, the transmission of the windows and the emission of plasma must be taken in account and pyroreflectometry has been presented as an alternative. Secondly, the characterization of the scene is performed. According to the literature, no major peek should appear for this kind of air plasma. The characterization of the quartz window revealed the probable pollution of the window during a previous experiment, so the quartz is not "grey". About the sample, the emissivity is the SiC's one for a new sample, and the Carbon's one for a used sample. The reflexion properties exhibit a strong directional effect but are compatible with a $45^{\circ}$ viewing angle. Finally, the experiment is performed and pyroreflectometry showed good first results. However, it has showed limitations which can be improved.

The first prospect is the adaptability to a higher thermal range. Indeed, the results presented here stops at $1500^{\circ} \mathrm{C}$ for radiance temperature, because the detectors started to saturate. Like reflectivity signals are quite weak, reducing the gain wouldn't be a good solution for those few-reflecting samples. The good compromise would be to reduce the filter's bandwidth, to lower thermal emission but not reflexion, which is currently under progress.

Then, the continuous pollution of the window is a real problem often encountered in vacuum facilities. A solution would be to install an inertial reference inside the chamber, which signal would be affected only by the pollution of the 
windows, so the transmission should drift with time. To sum up, an in-situ transmission measurement must be developed.

Finally, it would be interesting to visualize this scene with a camera, to take into account surface phenomenon. Pyroreflectometry knows a matrix extension called thermoreflectometry [10], which will be soon tested on the Plasmatron facility.

\section{REFERENCES}

[1] Hernandez D., "A concept to determine the true temperature of opaque materials using a tricolor pyroreflectometer". Review of Scientific Instruments, Volume 76, Issue 2, pp. 024904-024904-7 (2005).

[2] García, A.; Chazot, O.; Fletcher, D., "Investigations in Plasmatron Facilities on Catalicity Determination", Fourth Symposium on Aerothermodynamics for Space Vehicles: co-sponsored by European Space Agency. 2001, in Capua, Italy. Edited by R. A. Harris. European Space Agency, ESA SP-487, 2002. ISBN: 92-9092-789-5., p.489

[3] Balat-Pichelin M. ; Hernandez D. ; Olalde G. ; Rivoire B.; Robert J. F., :" Concentrated solar energy as a diagnostic tool to study materials under extreme conditions", Journal of Solar Engineering, vol.124,no3, pp.215222, (2002)

[4] Sakraker I., Asma C.O.,:"Experimental investigation of passive/active oxidation behavior of SiC based ceramic thermal protection materials exposed to high enthalpy plasma", Journal of the European Ceramic Society, Volume 33, Issue 2, Pages 351-359, (2013)

[5] Ph Bertrand, M Ignatiev, G Flamant, I Smurov, Pyrometry applications in thermal plasma processing, Vacuum, Volume 56, Issue 1, January 2000, Pages 71-76, ISSN 0042-207X, http://dx.doi.org/10.1016/S0042207X(99)00168-2

[6] Jean Thevenet, Monica Siroux, Bernard Desmet, Measurements of brake disc surface temperature and emissivity by two-color pyrometry, Applied Thermal Engineering, Volume 30, Issues 6-7, May 2010, Pages 753759, ISSN 1359-4311, http://dx.doi.org/10.1016/j.applthermaleng.2009.12.005

[7] Th. Duvaut, D. Georgeault, J.L. Beaudoin, Multiwavelength infrared pyrometry: optimization and computer simulations, Infrared Physics \& Technology, Volume 36, Issue 7, December 1995, Pages 1089-1103, ISSN 1350-4495, http://dx.doi.org/10.1016/1350-4495(95)00040-2

[8] C. Laux, R. Gessman, B. Hilbert, C. Kruger, Experimental study and modeling of infrared air plasma radiation, 30th Thermophysics Conference, June 1995

[9] Rousseau, B., Brun, J.F., Meneses, D.DeSousa, Echegut, P., Temperature Measurement: Christiansen Wavelength and Blackbody Reference, International Journal of Thermophysics, Volume 26, Issue 4, 2005.

[10] Sentenac T., Gilblas R., Hernandez D., Le Maoult Y., "Bi-color near infrared thermoreflectometry: A method for true temperature field measurement", Review of Scientific Instruments, Volume 83, Issue 12, pp. 124902124902-14 (2012) 